\title{
Open access? Widening access to Chimeric Antigen Receptor (CAR) therapy for ALL
}

\section{ABSTRACT}

T cells that are genetically modified to express chimeric antigen receptors (CARs) specific for CD19 show great promise for the treatment of relapsed/refractory acute lymphoblastic leukaemia (ALL). The first FDA approval of a cellular cancer therapy in 2017, i.e. Novartis' CD19-targeting CAR T cell product Kymriah ${ }^{\mathrm{TM}}$ within the context of relapsed/refractory paediatric ALL, followed rapidly by approval of Kite's Yescarta ${ }^{\mathrm{TM}}$ and more recently, Kymriah ${ }^{\mathrm{TM}}$ for diffuse large B cell indications in adults, highlighted the pace of progress made in this field.

In this review, we will consider the latest evidence from CAR T cell therapy for B lineage acute lymphoblastic leukaemia. We discuss the barriers to CAR T cell therapy for ALL patients and give a perspective on the strategy we have taken to date to widen access to CAR T cell therapy for UK paediatric patients with high risk ALL.

\section{Introduction}

Whilst standard therapy leads to a long-term event free survival in childhood ALL of approaching $90 \%{ }^{1,2}$, the outcomes of the $10 \%$ that relapse are much poorer with a predicted long term survival of $40-50 \%^{3-5}$. Within relapsed patients, high risk groups are identifiable by timing and site of relapse and have a long term event free survival of $30 \%$ or less even with allogeneic stem cell transplantation (allo-sct) ${ }^{3,5,6}$. The outcomes of those relapsing following allo-sct are dismal, with long term survival of only $10-20 \%$ in those undergoing second transplantation procedures ${ }^{7-9}$, which was also associated with significant adverse toxicity. For these groups, novel therapies were urgently needed, and access to CAR T cell therapy has been a "game-changer" in terms of outcomes.

Redirection of T cells by means of CAR expression enables potentially any cell surface moiety to be recognised in an HLA-independent manner. CARs can be engineered with a variety of ligand binding domains, most commonly antibody-derived single chain variable fragments (scFvs), but other antigen recognition domains, receptor ligands can also be employed. Whilst T cells have been used as the therapeutic effector cell in all of the large scale US studies of CD19 CAR targeting discussed in this review and summarised in Table 1, other effector cell populations for CAR therapy are actively being investigated. These include CAR engineered natural killer (NK) cells, cytotoxic immune cells which can potentially be infused as an off the shelf product, providing a short-lived therapeutic effect ${ }^{10}$ and cytokine induced killer (CIK) cells, derived from lymphocytes which acquire NK-like cytotoxic potential through the activation and culture process used to manufacture them ${ }^{11}$.

Targeting of CD19 by CAR T cells as therapy for B cell malignancies has served as a model for engineered cellular immunotherapy of cancer. A number of factors have contributed to the success of this approach, including restriction of CD19 expression within the haematopoietic system. Whilst B cell aplasia is an expected outcome of CD19 targeting, to date, there has been little evidence of significant infectious complications, suggesting that, at least in the 
short to medium term, the CD19+ B cell compartment is dispensable in treated patients. Immunoglobulin replacement has generally been given to those developing hypogammaglobulinaemia, but protective humoral immunity can persist despite persistent B cell aplasia, especially in adults ${ }^{12}$. Targeting of antigens restricted to the haematopoietic system may have advantages, not only for limiting off-tumour cellular damage, but may also modulate development of tolerance in antigen specific T cells ${ }^{13,14}$.

CD19 is expressed on a broad range of B cell malignancies, including $95 \%$ of B-lineage ALL, and because it forms part of the $B$ cell signal transduction complex, it is likely to contribute to $B$ cell survival, although CD19- tumour escape variants are a significant cause of therapeutic failure post CAR T cell therapy, as is discussed later. CD22 is also expressed on the majority of cells committed to the $B$ lineage ${ }^{15}$ and provides a basis for targeting of multiple B cell antigens with a view to reducing tumour antigenic escape.

A significant challenge to the haemato-oncological community is to now translate the lessons learnt from CD19 targeting to other haematological and solid organ malignancies, where, to date, CAR T cell therapies have been less successful.

\section{CAR T cell biology}

In most cases, chimeric antigen receptors link the antigen-specificity of a single chain variable fragment (scFv, derived from an antibody) with the downstream signalling machinery of a T cell (Figure 1) through incorporation of signalling domains derived from the CD3 zeta chain. A spacer region extends the antigen-binding domain from the cell membrane, and may be derived from a variety of molecules, e.g. IgG, CD8a, or CD28. The length and derivation of the spacer can be critical for CAR signalling ${ }^{16,17}$.

The modular design of CARs has facilitated incorporation of one or more co-stimulatory domains derived from receptors present naturally on $T$ cells and which mediate full activation of $T$ cells upon natural TCR signalling ${ }^{18,19}$. First generation CARs lacking a co-stimulatory domain (Figure 1) are capable of activating antigen-specific cytolysis, but such CAR T cells lack the ability to proliferate or generate cytokines in response to stimulation, which may contribute to failure to persist or expand adequately after transfer to recipients ${ }^{20,21}$. In other studies, however, first generation CAR T cells were noted to persist for more than a decade ${ }^{22}$, so other factors are also likely at play. By contrast, second and later generation CARs (containing one or more co-stimulatory domains, Figure 1 ) are capable of mediating full T cell activation, associated with expansion in the host of greater than a thousand fold ${ }^{23}$. In the setting of $B$ cell malignancies, particularly paediatric ALL, second generation, CD19-targeting CARs incorporating co-stimulatory domains derived from 4-1BB or CD28 been shown to be extremely efficacious at mediating long term remissions (Table 1 ).

\section{CAR T cell manufacture}

In general, CAR T cell products are made on a patient-specific basis, obtaining peripheral blood mononuclear cells (PBMCs) from the patient, or less commonly, their haematopoietic stem cell donor and using these as starting material for CAR T cell manufacture. PBMCs are obtained from whole blood, or more commonly via leucapheresis (density gradient centrifugation). Obtained PBMCs are then washed and activated using antibodies crosslinking CD3 and CD28 either in solution, associated with magnetic beads or colloidal matrices. 
Prior to activation, $T$ cells may be purified to reduce contaminating populations of granulocytes, monocytes and red cells which may improve the quality of the CAR T cell product generated. A further step, enhancing feasibility of CAR T cell manufacture where manufacturing slots are limiting, is cryopreservation of the leucapheresis product. This potentially allows $T$ cells to be collected at time-points prior to administration of chemotherapy which may detrimentally affect circulating $T$ cell populations.

Since CAR T cell products can vary widely in composition, particularly in CD4:CD8 ratio, and the proportion of cells retaining an early memory phenotype (central memory, naïve-like, stem cell memory), researchers at the Fred Hutchison Cancer Center/Seattle Children's Hospital have sought to pre-define CAR T cell populations infused either in terms of a 1:1 CD4:CD8 CAR T cell ratio ${ }^{24}$, or where available, a 1:1 ratio of CD8 central memory:bulk CD4 CAR T cells ${ }^{25}$. The studies ${ }^{24,} 25$ utilising these pre-defined CAR T cell populations have demonstrated highly favourable outcomes, discussed below. However, it remains unclear whether the additional manufacturing complexity involved, particularly the need for additional expansion, provides an efficacy advantage over unselected CAR T cell products.

Once activated, $T$ cells are then manipulated to express the CAR through a process of transduction either using viral, or non-viral gene delivery systems. The former require culture of packaging cell lines under GMP conditions for virus production, but have the advantage of very efficient, generally stable transduction of human T cells. Non-viral gene delivery via plasmids, nucleases or transposon-based technologies are cheaper, but efficiency is variable and cell viability can be compromised, depending on the methods used to introduce nucleic acids into therapeutic cells ${ }^{26}$.

Transduced cells are then expanded for a variable length of time to ensure an adequate dose is generated, before impurities are removed, and the cellular product sampled for quality tests. The CAR T cell product may be cryopreserved at this point to allow time for release assays to be performed prior to infusion into the patient. These assays include viability, stringent sterility tests, tests for purity of the therapeutic cell populations and assessments of transduction efficiency whether by quantitative PCR for sequences within the transgene or flow cytometry to detect the CAR or a tag introduced with the CAR.

Strategies have been developed to allow generation of 'universal' CAR T cells from a thirdparty donor. This overcomes the need to generate a bespoke product on a patient-specific basis, and means that a single PBMC donation can be used to generate 'off the shelf' CAR T cell doses for a number of patients. Additionally, it enables patients who are profoundly lymphopaenic and other subgroups (e.g. infant ALL) in whom it has been problematic to generate an autologous product to be treated with CAR T cells. However, as well as transduction with a transfer vector encoding the CAR, additional gene modifications are required to reduce the risk of graft versus host disease (GVHD) mediated by the therapeutic cells, with the potential for associated bone marrow aplasia or hepatic inflammation, as well as to prevent rejection of CAR T cells by the host immune system. At our centre, CD19 CAR T cells were generated from a healthy donor by lentivirally-mediated transduction with additional modifications including transcription activation-like effector nuclease (TALEN)mediated disruption of the genes encoding $T$ cell receptor alpha chain and CD52. The former modification reduces the risk of GVHD against the host and the latter renders the cells 
resistant to the effect of alemtuzumab, used as a transplant conditioning reagent, in turn promoting persistence of the modified cells. Such an approach for use of 'off-the-shelf' CAR T cells was reported in two infants relapsing after allo-SCT ${ }^{27}$, who remain in remission greater than 2 years out and the approach is now being investigated in the context of 2 UK studies in pediatric (NCT02808442) and adult patients (NCT02746952). Other groups have used manipulation of HLA class I to generate universal CAR T cells, with disruption of the HLA-I gene locus and enforced expression of non-classical HLA-I molecules such as HLA-E or G to prevent NK cell activation and rejection of such universal CAR T cells ${ }^{28}$.

\section{Second generation CAR T cells as therapy for ALL (see table 1) Efficacy}

3 major US centres have published studies of CD19 CAR T cell therapy for paediatric ALL. Investigators at the University of Pennsylvania conducted a phase 1-2a study of tisangenlecleucel (Kymriah ${ }^{\mathrm{TM}}$ ) in a cohort of 60 children and young adults demonstrating a complete remission rate of $93 \%$ and a 12 month relapse free survival of $55 \%{ }^{29}$. A phase 2 multicentre study of the same product is in progress and recently reported therapy of 75 patients with an $81 \%$ overall remission rate and $50 \%$ event free survival at 1 year. The median persistence of CAR T cells noted was approximately 6 months.

Lee and colleagues from the National Cancer Institute ${ }^{30}$ treated a cohort of 21 patients, defining a feasibility of $90 \%$ for generation of a CAR T cell product within the group. They defined a maximum tolerated dose of $1 \times 10^{6} \mathrm{CAR} \mathrm{T}$ cells $/ \mathrm{kg}$ patient weight. $60 \%$ of patients achieved an MRD negative remission and overall survival in this cohort was $52 \%$ at 10 months.

Investigators at the Seattle Children's Hospital reported outcomes with therapy of 45 children and young adults with a CD19CAR T cell product of defined composition (1:1 ratio of CD4:CD8 CAR T cells with high purity of CAR T cells). A 93\% MRD negative remission rate was achieved and estimated 12 month event free survival was $50.3 \%{ }^{24}$. Median duration of $B$ cell aplasia, as a correlate of CAR T cell persistence, was 3 months.

Results in adults ${ }^{25}, 31$ have been broadly similar. There are $80-90 \%$ response rates regardless of cytogenetic risk group. Long term event free survival rates are similar to those seen in children, of approximately $40-60 \%$. Across both paediatric and adult studies, there are differences in the proportions of patients consolidated with stem cell transplantation (SCT) at different centres, and this should be taken into account when assessing long term outcomes.

Relapse may be due to disease which continues to show CD19 expression, or arise from CD19negative variant clones. The former type of relapse is generally associated with failure of CAR $T$ cell persistence and recovery of normal $B$ cell populations, whereas the latter tends to arise in the ongoing presence of CAR T cells and results from the antigenic selection pressure exerted by them. Investigation of CD19- relapse has elucidated two main mechanisms by which CD19- variants may arise following therapy with T cells expressing the FMC63 CD19CAR which binds to exon 2 of CD19. This arises from various mechanisms, including due to alternatively spliced RNA isoforms which lack exon 2 or hemizygous deletion of this exon ${ }^{32}$. In some cases it does appear that relapse rates are higher with increased tumour burden ${ }^{31}$ which might simply reflect an increased chance of a CD19- clone emerging from a greater cell mass. 


\section{Toxicity}

There is a considerable burden of toxicity associated with CAR T cell therapy for ALL. In particular, these include cytokine release syndrome, CAR T cell related encephalopathy syndrome and B cell aplasia.

Whilst generally manageable with good supportive care, and, in the case of cytokine release syndrome, the judicious use of a specific agent i.e. the IL- 6 receptor antagonist tocilizumab; a few fatal cases of CAR T cell toxicity have occurred across adult studies utilising different CAR T cell platforms. In response to this, the US CARTOX Working Group was established to develop practice guidelines for monitoring, grading and management of these toxicities in adults $^{33}$. In general, the more severe manifestations of CAR-related toxicity occur in adults, and deaths have resulted from severe CRS or neurotoxicity.

\section{Cytokine release syndrome (CRS)}

This is an inflammatory syndrome, akin to sepsis, with variable severity in which patients present with fever, hypotension, hypoxia and potentially develop multi-organ failure. Particularly in children, the manifestations can overlap with haemophagocytic lymphohistiocytosis (HLH) resulting from immune-mediated macrophage activation. Risk factors for severe CRS include heavier disease burden and presence of co-morbidities. The onset generally occurs around the time of peak CAR T cell expansion, (days 2-7 post infusion). There is some influence of CAR design on the time to CRS, being faster with CD28 containing CARs than 4-1BB containing CARs, a trend which is mirrored by the times to peak CAR T cell expansion. Most patients will develop some degree of CRS symptoms, however there is an incidence of severe (grade 3 or greater) CRS in $23-46 \% 24,30,34$, for which patients require management in an intensive care setting.

Various groups have studied biomarkers to predict severe $\mathrm{CRS}^{35}, 36$. In general, these have included high serum levels of pro-inflammatory cytokines e.g. IL-6, IFN $\gamma$, as well as of antiinflammatory cytokines which are induced in concert with these e.g. IL-10. However, in general, the predictive value of such biomarker signatures is study-specific and may relate to differences in CAR T cell products or the patient population studied. Severity of CRS has correlated with disease burden, peak of plasma/serum IL-6 ${ }^{30}, 35,37$, earlier onset of CRS $^{35}, 38$ and CAR T cell dose $24,25,30,38$ in a number of studies. This latter finding has led to adoption of split $^{39}$ or risk adapted CAR T cell dosing ${ }^{25}$ to mitigate risk of severe CRS. This is the strategy adopted in a study at our centre (the AMELIA study, EudraCT 2016-004680-39), in which a proportion of the total CAR T cell dose (30\%) is delivered initially, with the rest of the dose being administered 5-10 days later, contingent upon absence of development of severe toxicity up to this point.

In view of the relationship between disease burden and CRS severity, another strategy to reduce the risk of severe CRS is delivery a cycle of chemotherapy with the aim of debulking disease where possible prior to lymphodepletion. This is not always achievable with highly refractory patients, however, and repeated chemotherapy cycles may impact CAR T cell fitness, thus limiting CAR T cell efficacy. At our centre, we took a different approach to mitigating toxicity. The role of the greater affinity of CAR T cell interactions in mediating greater toxicity compared to TCR engineered CAR T cells has not been widely explored. By selecting CARs with defined binding kinetics and affinities lower than those which have 
achieved marketing authorization, it may be possible to obtain therapeutic efficacy with an improved toxicity profile. We have developed a CAR with a lower affinity for CD19 contributed to by a faster off-rate but equivalent on-rate to the CD19 binder present in Kymriah (FMC63) in order to investigate the utility of this approach in reducing CAR-related toxicity ${ }^{40}$.

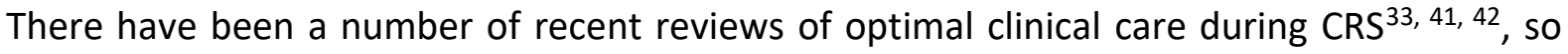
treatment protocols are not considered in detail here. It must be mentioned though that there are differing published grading systems and management guidelines for CRS. It is therefore important to agree on a given protocol at each centre delivering CAR T cell therapy, to ensure consistency in management of this complication. Important differential diagnoses, namely sepsis, should be managed presumptively until excluded by appropriate investigations.

Current best practice includes closely-monitored supportive therapy e.g. pre-emptive fluid management, inotrope support, as well as with IL- 6 axis blockade upon development of grade 2 CRS. Tocilizumab is a monoclonal antibody blocking the IL-6 receptor which is licensed for management of CRS as well as inflammatory arthritides in children. Siltuximab, a monoclonal antibody which binds and neutralises IL-6, is licensed for multicentric Castleman's disease. Both these are effective therapies for CRS, inducing rapid reversal of CRS symptoms in most patients. Use of tocilizumab with more recent CRS management protocols is required in 25$40 \%^{24,25,31,34}$.

Short courses of steroids may be added if symptoms persist after tocilizumab administration, or repeated IL-6 blockade may be necessary. To date, there appears to be little impact of tocilizumab on CAR T cell expansion, response rates or durability of response at least on the basis of modelling CAR T cell kinetics in patients with severe $\mathrm{CRS}^{43}$, but whether this also holds for patients with lower CRS severity has not been systematically investigated. Since cytokine storm involves elevated circulating levels of a number of pro-inflammatory mediators, it is likely that novel therapeutic options will be developed for those failing to respond to tocilizumab and steroids ${ }^{44}$.

Earlier implementation of IL-6 blockade (e.g. at grade 1 CRS) may be considered in the setting of significant co-morbidities. During the peak of CRS, vigilance is required for development of further complications, including CRES, cardiac ventricular dysfunction, cardiac arrhythmias, haemophagocytic lymphohistiocytosis and endothelial activation through appropriate baseline measurements (e.g. cognitive assessment, EEG, neuroimaging, echocardiography, electrocardiographic telemetry, ferritin, fibrinogen, d-dimers, respectively) and ongoing monitoring of these parameters if changes subsequently develop.

More recently, an association between severe CRS and coagulopathy / endothelial activation has been highlighted. Investigation of biomarkers of severe CRS in a predominantly paediatric cohort revealed an association between low fibrinogen and grade 4 CRS in paediatric patients ${ }^{35}$. This was explored further in an adult cohort treated at the Fred Hutchinson Cancer Center ${ }^{38,45}$ where grade 4 or greater CRS was associated with hypofibrinogenaemia, increased $\mathrm{d}$-dimers and reduced platelets, consistent with disseminated intravascular coagulation.

\section{CAR T cell-related encephalopathy syndrome (CRES)}


Patients with CRES appear encephalopathic with a variety of presenting symptoms including dysphasia, dysgraphia, obtundation, and seizures. The frequency of seizures varies dependent on the CAR design, being more frequent in studies where a CD28-containing CAR was adopted. Symptoms may be of variable severity, however, severe neurotoxicity generally develops in those with more severe $\mathrm{CRS}^{24,38}$ and often develops after the peak of CRS manifestations ${ }^{38}$. The symptoms of CRES overlap with those of infective encephalopathy as well as neurological toxicity resulting from methotrexate or fludarabine administration, and these are relevant differentials to consider. Neuro-imaging and CSF analysis can be highly informative in this regard, with flow cytometry on CSF to demonstrate the nature of any cell populations identified. Demonstration of white matter change is more likely to be associated with drug-related encephalopathy and is generally absent in those with CRES ${ }^{33}$.

The mechanism for neurotoxicity is not clearly understood, but in view of the correlation between severity of CRS and of CRES, the latter does appear to be related to systemic inflammation in the setting of rapid early CAR T cell expansion, and particularly with the rapid upstroke of systemic inflammatory cytokines such as IL-6 $6^{38}$. This has led to the implication of rapid transfer of inflammatory cytokines from the systemic circulation into the CSF as a potential mechanism. Further, delineation of endothelial activation in severe neurotoxicity ${ }^{45}$ may allow rational drug targeting to ameliorate CRES.

Unfortunately, in some cases, severe neurotoxicity can have a fatal outcome, such as was the case in the development of JCAR015 by Juno therapeutics. A total of 5 fatal cases of neurotoxicity resulting from cerebral oedema led to the closure of this study. Whilst it was asserted that early rapid CAR T cell expansion related to the presence of a CD28-derived costimulatory domain may have contributed to the severity of neurotoxicity seen, in reality, it is likely that a number of factors contributed. Indeed, fatal neurotoxicity was reported in $3 \%$ of adults treated with a 4-1BB domain containing CAR for a variety of B cell malignancies at the Fred Hutchison Cancer Centre in Seattle ${ }^{45}$.. There is great interest in determining a biomarker profile capable of predicting high risk neurotoxicity and in the same cohort of patients, a combination of fever $\geq 38.9^{\circ} \mathrm{C}$ with IL- 6 levels of $>16 \mathrm{pg} / \mu \mathrm{L}$ and monocyte chemoattractant protein (MCP) $1>1344 \mathrm{pg} / \mu \mathrm{L}$ in the first 36 hours post CAR T cell infusion was associated with a very high risk of severe neurotoxicity ${ }^{45}$.

An association has been noted between higher CRS and neurotoxicity grades and serum Von Willebrand Factor (VWF), suggesting greater endothelial activation in both CRS and CRES and

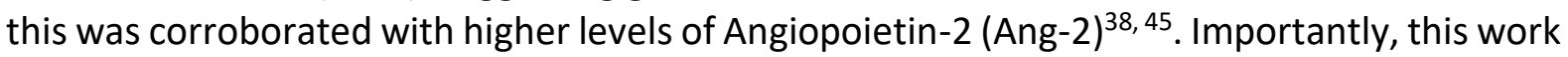
also demonstrated that pre-existing endothelial activation (prior to CAR T cell infusion, as determined by increased VWF and Ang-2:Ang-1 ratios), may be associated with increased subsequent CRS severity. Theoretically, then, pharmacological manipulation of endothelial activation may modify CRS severity, providing potential interventions to ameliorate CRS.

There is no specific management of CRES, unless it exists concomitantly with CRS, in which case tocilizumab therapy is indicated as above. Siltuximab may also be administered. Whilst there are less data available on the use of this latter agent, its mode of action in directly neutralising circulating IL- 6 is theoretically advantageous over tocilizumab, because serum IL6 levels often increase post tocilizumab-mediated IL-6R blockade, and may contribute to increased CRES severity. However, if encephalopathy develops after CRS, then short course 
steroids are often considered as first line therapy. The delineation of novel mediators implicated in CRES such as increased VWF and Ang-2 may provide rational targets for novel CRES therapies in the future.

\section{B cell aplasia}

Rational targeting of the CD19+ B cell compartment will result in B cell aplasia, with the possibility of hypogammaglobulinaemia if B cell aplasia is persistent. In our centre, if hypogammaglobulinaemia is persistent following CAR $T$ cell infusion, prophylactic immunoglobulin replacement is instituted. However, despite the sometimes profound B cell aplasia sustained post CAR T cell infusion, the risk of severe infections are low, and seem to be associated with development of severe $\mathrm{CRS}^{46}$.

\section{Cytopenias}

Cytopenias following CAR T cell therapy are common and are contributed to by a number of factors such as intensity of lymphodepletion, number of prior therapy lines, disease burden going into CAR T cell therapy and graft function post allo-SCT. For example, patients at our centre receiving a combination of $1.5 \mathrm{~g} / \mathrm{m}^{2}$ cyclophosphamide and $150 \mathrm{mg} / \mathrm{m}^{2}$ fludarabine as lymphodepletion are typically cytopenic for at least 28 days whereas those undergoing less intensive lymphodepletion $\left(0.9 \mathrm{~g} / \mathrm{m}^{2}\right.$ cyclophosphamide and $90 \mathrm{mg} / \mathrm{m}^{2}$ fludarabine) tend to be cytopaenic for about half this interval (Amrolia and Ghorashian, unpublished results).

These intervals are not unusual within the field, for example, $15 \%$ of patients treated with CD19 CAR T cell therapy for a range of relapsed/refractory B cell malignancies were neutropenic beyond 28 days post CAR T cell therapy ${ }^{46}$, and $40-50 \%$ of patients treated with Tisangenlecleucel/Kymriah ${ }^{\mathrm{TM}}$ demonstrated grade 3-4 cytopenias which persisted beyond 28 days ${ }^{34}$. Further, this degree of cytopenia is not restricted to therapy with CD19-targeting CARs, and is also seen for example, in patients receiving CD22-directed CAR T cell therapy ${ }^{47}$. These data suggest it may be a cytokine-mediated, rather than antigen-mediated phenomenon.

Whilst contributing to the complexity of managing these patients, and potentially compounding infection risk in patients who might also have hypogammaglobulinaemia, the overall risk of life-threatening infections in relapsed/refractory patients managed with these therapies is low, as mentioned above at $3-5 \%^{34,46}$.

\section{Barriers to CAR T cell therapy}

Barriers to CAR T cell therapy exist at all stages of implementation of these cellular therapeutics. These are discussed below.

\section{CAR T Cell Manufacture}

Manufacturing CAR T cells is a complex process performed under GMP conditions and represents a key barrier to broader application of this technology. Initially, manufacturing protocols facilitated high levels of $T$ cell expansion through use of high dose IL-2 with prolonged ex vivo culture post transduction. More recent recognition of the importance of retaining of T cells within an early differentiation state during CAR T cell manufacture ${ }^{48-52}$ has led to minimising the expansion phase required, so that the total process generally lasts 7-10 days. A further limitation to manufacturing capacity incudes the complexity of the 
manufacturing process involved, particularly the number of manual processing steps required. Use of semi-automated, closed system manufacturing platforms such as the Miltenyi Prodigy has been demonstrated to significantly reduce man hours required for CAR $T$ cell manufacture ${ }^{53}$ and to robustly generate CAR $T$ cell products suitable for clinical studies ${ }^{54}$. As such, generation of CAR T cell products for licensed indications beyond B cell malignancies seems more feasible, though this rests on the flexibility of the automated manufacturing platform employed and the governance issues in providing industry standards for widely diverse processes.

Manufacturing failures are noted, depending on the manufacturing methods and the dose required, but are reported at approximately $10 \%^{30,34}$. There are many factors which may have a bearing, for example, the nature of anti-leukaemic therapy patients undergo prior to leucapheresis. Certain chemotherapy agents in particular, e.g. cyclophosphamide and cytarabine may be responsible for depleting $T$ cells from early memory subsets that particularly seem to contribute to expansion ${ }^{55}$. By selecting cells from these subsets and then employing expansion steps, it may be possible to eliminate manufacturing failures, as has been the case in some studies ${ }^{24,25}$.

\section{Vectors}

As discussed above, newer methods of gene transduction may obviate the need to use viral vectors. Whilst efficient, viral vectors require another costly GMP process for their production prior to incorporation in CAR T cell manufacture. Different studies have incorporated either lenti or retroviral gene transduction methods (see Table 1), giving comparable initial response rates, but at present, it is not clear whether one approach is better than the other because studies have also utilised different CAR design and manufacturing methods.

Unlike retroviral vectors, production of clinical grade lentiviral vector has, to date, involved transient transfection of human embryonic kidney (HEK) 293T cells. This allows only limited batches of lentivirus to be generated on a one-off basis and with a potential for significant batch-to-batch variability in titre, leading in turn to variability in CAR T cell manufacture. By contrast, use of stable producer cell lines for retroviral vector production leads to a highly scalable process, which is reliable and reproducible producing high yields of viral titre.

However, increasing recognition that differences in the integration profile of retrovirally and lentivirally transduced CAR $T$ cells leads to potentially deleterious differences in CAR expression in terms of the fitness of CAR T cells generated. This would suggest that, for a given CAR design and targeted tumour antigen, these transduction platforms may not be equivalent in terms of the quality of CAR T cell product generated ${ }^{56,57}$. Whilst much research effort is focussed on generating lentiviral producer cell lines for clinical purposes ${ }^{58,59}$, at present, viral yields generally remain lower than those generated by transient transfection.

Non-viral transduction methods have been employed in CD19 CAR T cell studies. These include transposon-based gene transduction such as the sleeping beauty system which facilitate stable transgene expression, have proven feasible for generation of clinical CAR T cell products for patients and are significantly less costly than use of viral transduction ${ }^{26}$. Further advantages include a near-random integration pattern, rather than preferential targeting of highly expressed genes, as is seen with viral transduction methods. There is a 
concern that dysregulated gene expression caused by non-random transgene insertion may lead to oncogenic potential, such as those seen following gene-engineering of haematopoietic stem cells ${ }^{60,61}$, though in practice, there have been no cases of oncogenesis of this type with transduction of mature $T$ cells across CAR T cell or other $T$ cell studies across the globe. Moreover, the cell manipulation and prolonged expansion used with non-viral transduction may impact on the biological fitness of CAR T cells, though prolonged persistence has been documented in early clinical studies of CD19 CAR T cell therapy in the setting of adjunctive allogeneic or autologous stem cell transplantation ${ }^{26}$. Data on disease-related outcomes from these studies are eagerly awaited in order to understand whether their therapeutic efficacy is equivalent to virally-transduced CAR T cells.

\section{Controlling disease prior to manufacture - a difficult balance}

In any patient being considered for CAR T cell therapy, there is a need to co-ordinate therapy required to control disease burden with the requirement for a decent lymphocyte on given leucapheresis date to facilitate CAR T cell manufacture, particularly if manufacturing slots are limited. The optimal time is for leucapheresis is unclear - too early post relapse and the blast percentage may be high, with risk of transduction and subsequent expansion of CARtransduced ALL blasts in the recipient ${ }^{62}$. However, the quality of the CAR T cell product can be affected by prior chemotherapy. For example, certain agents such as cytarabine and cyclophosphamide may have a demonstrable impact on subsequent CAR T cell fitness ${ }^{55}$. Whilst other novel agents e.g. blinatumomab or inotuzumab may also be utilised, it is unclear if this additional selection pressure prior to CAR T cell therapy may compound or mitigate the risk of relapse with $\mathrm{CD} 19$ - escape variants respectively. Further, an optimal interval from last chemotherapy is required. Whilst leucapheresis of lymphopaenic patients is feasible, contaminating populations within the PBMC pool obtained can cause significant issues by consumption of e.g. cytokines, activating beads, such that measures to remove monocytes and neutrophils e.g. elutriation, $T$ cell selection or plastic adherence ${ }^{63}$ can improve the feasibility of generating an effective CAR T cell dose in all patients. Optimisation of relapse protocols incorporating CAR T cell therapy is required.

If the patient has already undergone allo-SCT, a feasible option is to generate donor-derived CAR T cells. This has the advantage of overcoming lymphopaenia or intrinsic $T$ cell deficits in the host, and allows CAR T cell manufacture to occur independently of managing the disease itself, of particular benefit for those with aggressive disease. Several studies, including one at our institution ${ }^{21}$, have highlighted the feasibility and safety of such approach, since, at least with CD28-containing CAR constructs and in the absence of lymphodepleting conditioning, the incidence of acute GVHD is low ${ }^{64,65}$. In one study utilising a 4-1BB containing CAR, both patients treated with donor-derived CAR T cell therapy for ALL developed grade 2 acute GVHD (skin and liver) ${ }^{66}$. Whilst further evaluation in the context of larger clinical studies is warranted, pre-clinical evidence supports the induction of exhaustion and activation induced death of allogeneic CD19CAR T cells containing a CD28-containing domain, which abrogates their ability to mediate GVHD. This was not the case after transfer of 4-1BB containing CD19 CAR T cells, which were capable of mediating GVHD in the same model system ${ }^{67}$.

Manufacturing feasibility is a significant consideration in CAR T cell therapy for infant ALL. Both the pace of disease may not allow for prolonged intervals between chemotherapy to allow adequate lymphocyte recovery and because it appears technically difficult to generate 
CAR T cells from such patients. It is unclear if this difficulty reflects differences in the biology of infant $T$ cells or the increased intensity of chemotherapy given in this disease setting. A further challenge facing this population are the frequent association with rearrangement of the MLL gene ${ }^{68}$ which provides a particular predilection to evolution of a myeloid leukaemic clone leading to subsequent myeloid leukaemic relapse ${ }^{69}$. As such, therapy of infant ALL remains an ongoing area of unmet clinical need and represents an ideal setting in which to test universal CAR T cell approaches. Here, generation of fixed batches of universal CAR T cells from a third party donor allows an off-the-shelf product, as discussed above.

\section{Preventing relapse post CAR T cell therapy}

Since relapses of ALL are contributed to by failure of persistence of CAR T cells, with recovery of normal B cells often heralding relapse, usually with CD19+ disease, several groups have investigated the utility of re-dosing with CAR T cells. In general, this approach has not been effective in prolonging CAR T cell persistence, and in some cases, a T cell mediated immune response against recipient CAR T cells have been identified as mediating poor persistence ${ }^{25}$. Since long-lived T cells capable of mediated immunosurveillance have been identified as being derived from early memory phenotype cells as discussed above, CAR T cell manufacturing methods are currently generally designed with the aim of preserving these populations as far as possible. Further efforts to improve outcomes by optimising CAR T cell persistence have led to studies utilising CARs which have been humanised to minimise anti-CAR immune responses, with favourable disease-related outcomes ${ }^{70}$, even in patients who have been refractory to standard CAR T cell therapy.

Finally, in an attempt to circumvent antigenic escape through selection of CD19- escape variants, groups have investigated the use of other targets for CAR T cells such as CD22 ${ }^{71}$. The utility of this approach was demonstrated, with responses seen in patients having failed prior CD19 targeting CAR T cell therapy, however, CD22 was down-regulated in 7 of 8 patients who subsequently relapsed, suggesting that single agent targeting, regardless of the antigen targeted, has the potential for creating antigen escape variants. Thus, multi-antigenic targeting, for example with CARs targeting CD19 and CD22 is the subject of a number of studies, including at our centre (the AMELIA study, EudraCT 2016-004680-39) and $\mathrm{NIH}^{72}$.

\section{Developing an integrated pathway for CAR T cell therapy: UK approach}

At our centre, we currently have a number of CD19 CAR T cell studies open (the CARPALL study NCT02443831, UCART19 NCT02808442, the AMELIA study, EudraCT 2016-004680-39). By having a comprehensive programme of CAR T cell studies, we are able to offer CAR T cell therapies to a broader range of patients. Patients are identified from a national referral network, promoting equity of access of these therapies to patients across the UK. Patients in whom an autologous product is unlikely to be generated e.g severe lymphopaenia, infant ALL, are treated with universal CAR T cells. The AMELIA study, of CD19 and CD22 directed CAR T cell therapy, offers an option for those with CD19 negative disease.

Manufacturing capacity for this programme is shared across academic (UCL - the CARPALL study) and industrial partners (Autolus - the AMELIA study, Pfizer - UCART19), ensuring we are able to treat up to 2-3 patients per month. 
Management of relevant toxicities is carried out with an experienced medical team and in close discussion with the PICU staff. Patients are flagged prior to CAR T infusion to facilitate timely transfer to higher dependency care. Clear protocols govern management of both CRS and neurotoxicity ensures patients are managed in a consistent fashion.

\section{Conclusion: broadening access and future challenges.}

Since Eshhar et $\mathrm{al}^{73}$. delineated the use of modular chimeric immunoreceptors leading to the development of CARs 15 years ago, progress towards their therapeutic potential has been rapid. Gilead's recent acquisition of Kite Pharma, and subsequently, Celgene's acquisition of Juno reflects the drive for pharma giants to position themselves favourably within the developing CAR T cell therapy market. Until European licenses are granted, however, access to CAR T cell therapy in Europe currently remains restricted to those eligible for CAR T cell studies.

Through the establishment of multiple partners in delivering these complex studies of engineered cellular therapeutics, we hope we have gone some way to provide UK access to the promise of their therapeutic efficacy. However, looking forward to the goals for the next 15 years, multiple challenges remain, namely, prevention of CD19 negative relapse where this antigen alone is targeted, scaling up delivery of this therapy to all eligible patients and broadening the indications for its use. 
First generation CAR

A

B

C

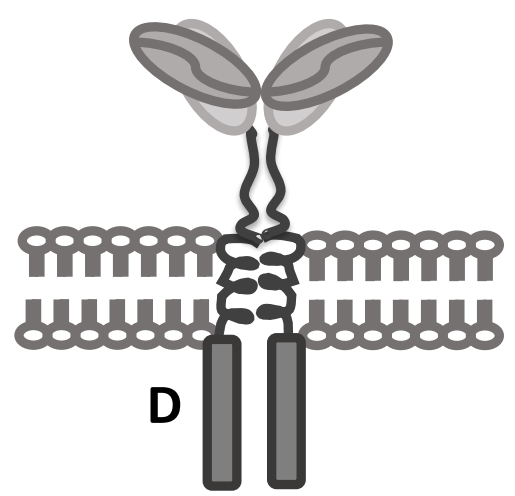

Second generation CAR

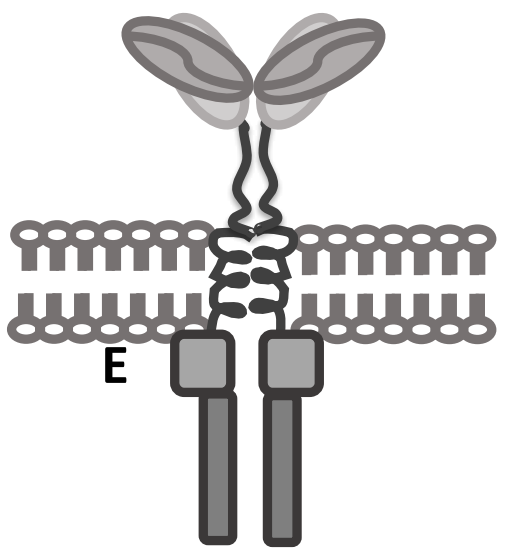

Third generation CAR

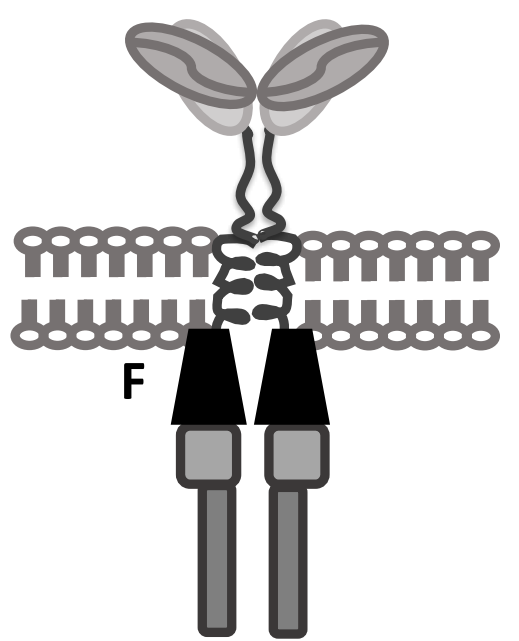

Figure 1. The Anatomy of a CAR.

$\mathrm{A}=$ ligand binding domain, most commonly derived from an scFv, B = spacer or stalk region, derived from a variety of molecules e.g. IgG Fc domain, $C D 8, C=$ transmembrane region, commonly derived from the same molecule as the membrane-proximate endodomain, $D=$ signaling domain, usually derived from CD3 zeta chain, $E, F=$ co-stimulatory endodomains - may be derived from a variety of costimulatory molecules e.g. CD28, 4-1BB, OX40, and may be multiplexed within third generation CARs. 
Table 1 Clinical studies of CAR T cell therapy for ALL.

ING - information not given, LCLs - lymphoblastoid B-cell lines

\begin{tabular}{|c|c|c|c|c|c|c|c|c|c|c|c|}
\hline Site/Sponsor & $\mathrm{n}$ & Target/Binder & $\begin{array}{l}\text { Co- } \\
\text { stimulatory } \\
\text { domain }\end{array}$ & Viral vector & $\begin{array}{l}\text { Pre- } \\
\text { selection }\end{array}$ & Activation & $\begin{array}{l}\mathrm{CR} \\
\text { rate }\end{array}$ & $\begin{array}{l}\text { MRD } \\
\text { neg } \\
\text { CR } \\
\text { rate } \\
\end{array}$ & $\begin{array}{l}\text { Grade 3- } \\
5 \\
\text { CRS }\end{array}$ & $\begin{array}{l}\text { Grade } 3-5 \\
\text { neurotoxicity }\end{array}$ & $\begin{array}{l}\text { Allo-SCT as } \\
\text { adjunctive } \\
\text { therapy }\end{array}$ \\
\hline $\begin{array}{l}\text { Baylor College of } \\
\text { Medicine }^{65}\end{array}$ & $\begin{array}{l}4 \\
\mathrm{ALL}\end{array}$ & CD19/FMC63 & CD28 & Retrovirus & $\begin{array}{l}\text { Virus } \\
\text { specific } \\
\text { CTL }\end{array}$ & $\begin{array}{l}\text { Autologous } \\
\text { LCLs }\end{array}$ & $75 \%$ & ING & $0 \%$ & ING & $\begin{array}{l}\text { Treated 3-12 } \\
\text { months post } \\
\text { allo-SCT }\end{array}$ \\
\hline MSKCC $^{31,74}$ & 53 & CD19/ SJ25C1 & CD28 & Retrovirus & None & $\begin{array}{l}\text { CD3/D28 } \\
\text { beads }\end{array}$ & $83 \%$ & $56 \%$ & $26 \%$ & $42 \%$ & $39 \%$ \\
\hline CHOP/UPenn ${ }^{29,37}$ & 75 & CD19/ FMC63 & $4-1 B B$ & Lentivirus & None & $\begin{array}{l}\text { CD3/D28 } \\
\text { beads }\end{array}$ & $93 \%$ & $88 \%$ & $27 \%$ & ING & $10 \%^{37}$ \\
\hline $\begin{array}{l}\mathrm{NCl} \\
30,75\end{array}$ & 51 & CD19/ FMC63 & CD28 & Retrovirus & None & $\begin{array}{l}\text { CD3/D28 } \\
\text { beads }\end{array}$ & $60.8 \%$ & $55 \%$ & $13.5 \%$ & $5 \%^{30}$ & $40 \%$ \\
\hline $\begin{array}{l}\text { Chinese PLA General } \\
\text { Hospital }^{76}\end{array}$ & $\begin{array}{l}8 \\
\text { ALL }\end{array}$ & CD19/ FMC63 & $4-1 B B$ & Lentivirus & None & $\begin{array}{l}\text { CD3 } \\
\text { antibody }\end{array}$ & $50 \%$ & $25 \%$ & $25 \%$ & $0 \%$ & ING \\
\hline $\mathrm{NCl}^{64}$ & $\begin{array}{l}5 \\
\mathrm{ALL} \\
\end{array}$ & CD19/ FMC63 & CD28 & Retrovirus & None & $\begin{array}{l}\text { CD3 } \\
\text { antibody }\end{array}$ & $80 \%$ & $80 \%$ & $100 \%$ & $40 \%$ (headache) & $20 \%$ \\
\hline FHCRC $^{25}$ & 32 & CD19/ FMC63 & $4-1 B B$ & Lentivirus & $\begin{array}{l}\text { CD8 or } \\
\text { CD8Tcm + } \\
\text { CD4 }\end{array}$ & $\begin{array}{l}\text { CD3/D28 } \\
\text { beads }\end{array}$ & $100 \%$ & $93 \%$ & $\begin{array}{l}23 \% \\
\text { (require } \\
\text { ITU) }\end{array}$ & $50 \%$ & $43 \%$ \\
\hline MDACC $^{77}$ & $\begin{array}{l}17 \\
\mathrm{ALL}\end{array}$ & CD19/ING & CD28 & $\begin{array}{l}\text { Sleeping } \\
\text { beauty } \\
\text { transposon }\end{array}$ & None & None & $53 \%$ & ING & $0 \%$ & $0 \%$ & $100 \%$ \\
\hline Seattle Childrens ${ }^{24}$ & 45 & CD19/ FMC63 & $4-1 B B$ & Lentivirus & $\mathrm{CD} 8+\mathrm{CD} 4$ & $\begin{array}{l}\text { CD3/D28 } \\
\text { beads }\end{array}$ & ING & $93 \%$ & $23 \%$ & $21 \%$ & $26 \%$ \\
\hline $\mathrm{CHOP}^{70}$ & 36 & $\begin{array}{l}\text { CD19/ ING, } \\
\text { humanised }\end{array}$ & $4-1 B B$ & Lentivirus & None & $\begin{array}{l}\text { CD3/D28 } \\
\text { beads }\end{array}$ & $83 \%$ & ING & $13 \%$ & ING & ING \\
\hline $\mathrm{UCL}^{21}$ & 11 & CD19/FMC63 & $\begin{array}{l}\text { First } \\
\text { generation }\end{array}$ & Retrovirus & EBV CTL & $\begin{array}{l}\text { Autologous } \\
\text { LCLs }\end{array}$ & $45 \%$ & $45 \%$ & $0 \%$ & $0 \%$ & $100 \%$ \\
\hline $\mathrm{NCl}^{72}$ & 21 & $\begin{array}{l}\text { CD22 / m971 } \\
\text { human }\end{array}$ & $4-1 B B$ & Lentivirus & CD3+ & $\begin{array}{l}\text { CD3/D28 } \\
\text { beads }\end{array}$ & $57 \%$ & $43 \%$ & $0 \%$ & $0 \%$ & ING \\
\hline Novartis $^{34}$ & 75 & CD19/ FMC63 & $4-1 B B$ & Lentivirus & None & $\begin{array}{l}\text { CD3/D28 } \\
\text { beads }\end{array}$ & $81 \%$ & $81 \%$ & $46 \%$ & $13 \%$ & $11 \%$ \\
\hline
\end{tabular}




\section{References}

1. Vora A, Goulden N, Wade R et al. Treatment reduction for children and young adults with low-risk acute lymphoblastic leukaemia defined by minimal residual disease (UKALL 2003): a randomised controlled trial. Lancet Oncol. 2013;14 (3):199-209.

2. Pui CH, Yang JJ, Hunger SP et al. Childhood Acute Lymphoblastic Leukemia: Progress Through Collaboration. J Clin Oncol. 2015;33 (27):2938-2948.

3. Roy A, Cargill A, Love $S$ et al. Outcome after first relapse in childhood acute lymphoblastic leukaemia - lessons from the United Kingdom R2 trial. Br J Haematol. 2005;130 (1):67-75.

4. Parker C, Waters R, Leighton $C$ et al. Effect of mitoxantrone on outcome of children with first relapse of acute lymphoblastic leukaemia (ALL R3): an open-label randomised trial. Lancet. 2010;376 (9757):2009-2017.

5. Tallen G, Ratei R, Mann G et al. Long-term outcome in children with relapsed acute lymphoblastic leukemia after time-point and site-of-relapse stratification and intensified short-course multidrug chemotherapy: results of trial ALL-REZ BFM 90. J Clin Oncol. 2010;28 (14):2339-2347.

6. Freyer DR, Devidas $M, L a$ et al. Postrelapse survival in childhood acute lymphoblastic leukemia is independent of initial treatment intensity: a report from the Children's Oncology Group. Blood. 2011;117 (11):3010-3015.

7. Saarinen-Pihkala UM, Heilmann C, Winiarski J et al. Pathways through relapses and deaths of children with acute lymphoblastic leukemia: role of allogeneic stem-cell transplantation in Nordic data. J Clin Oncol. 2006;24 (36):5750-5762.

8. Bajwa R, Schechter T, Soni S et al. Outcome of children who experience disease relapse following allogeneic hematopoietic SCT for hematologic malignancies. Bone Marrow Transplant. 2013;48 (5):661-665.

9. Poon LM, Bassett RJ, Rondon $\mathrm{G}$ et al. Outcomes of second allogeneic hematopoietic stem cell transplantation for patients with acute lymphoblastic leukemia. Bone Marrow Transplant. 2013;48 (5):666-670.

10. Rezvani K, Rouce R, Liu E, Shpall E. Engineering Natural Killer Cells for Cancer Immunotherapy. Mol Ther. 2017;25 (8):1769-1781.

11. Magnani CF, Turazzi N, Benedicenti F et al. Immunotherapy of acute leukemia by chimeric antigen receptor-modified lymphocytes using an improved Sleeping Beauty transposon platform. Oncotarget. 2016;7 (32):51581-51597.

12. Bhoj VG, Arhontoulis D, Wertheim G et al. Persistence of long-lived plasma cells and humoral immunity in individuals responding to CD19-directed CAR T cell therapy. Blood. 2016 13. Flutter B, Edwards N, Fallah-Arani F et al. Nonhematopoietic antigen blocks memory programming of alloreactive CD8 $+\mathrm{T}$ cells and drives their eventual exhaustion in mouse models of bone marrow transplantation. J Clin Invest. 2010;120 (11):3855-3868.

14. Ghorashian S, Veliça P, Chua I et al. CD8 T cell tolerance to a tumor-associated selfantigen is reversed by CD4 T cells engineered to express the same T cell receptor. J Immunol. 2015;194 (3):1080-1089.

15. Shah NN, Stevenson MS, Yuan CM et al. Characterization of CD22 expression in acute lymphoblastic leukemia. Pediatr Blood Cancer. 2015;62 (6):964-969.

16. James SE, Greenberg PD, Jensen MC et al. Antigen sensitivity of CD22-specific chimeric TCR is modulated by target epitope distance from the cell membrane. J Immunol. 2008;180 (10):7028-7038. 
17. Hudecek M, Sommermeyer D, Kosasih PL et al. The nonsignaling extracellular spacer domain of chimeric antigen receptors is decisive for in vivo antitumor activity. Cancer Immunol Res. 2015;3 (2):125-135.

18. Finney HM, Lawson AD, Bebbington CR, Weir AN. Chimeric receptors providing both primary and costimulatory signaling in T cells from a single gene product. J Immunol. 1998;161 (6):2791-2797.

19. Maher J, Brentjens RJ, Gunset G, Riviere I, Sadelain M. Human T-lymphocyte cytotoxicity and proliferation directed by a single chimeric TCRzeta /CD28 receptor. Nat Biotechnol. 2002;20 (1):70-75.

20. Savoldo B, Ramos CA, Liu E et al. CD28 costimulation improves expansion and persistence of chimeric antigen receptor-modified T cells in lymphoma patients. J Clin Invest. 2011;121 (5):1822-1826.

21. Rossig C, Pule M, Altvater B et al. Vaccination to improve the persistence of CD19CAR gene-modified T cells in relapsed pediatric acute lymphoblastic leukemia. Leukemia. 2017;31 (5):1087-1095.

22. Scholler J, Brady TL, Binder-Scholl $G$ et al. Decade-long safety and function of retroviral-modified chimeric antigen receptor T cells. Sci Transl Med. 2012;4 (132):132ra53.

23. Grupp SA, Kalos M, Barrett $D$ et al. Chimeric antigen receptor-modified T cells for acute lymphoid leukemia. N Engl J Med. 2013;368 (16):1509-1518.

24. Gardner RA, Finney O, Annesley C et al. Intent to treat leukemia remission by CD19CAR T cells of defined formulation and dose in children and young adults. Blood. 2017

25. Turtle CJ, Hanafi LA, Berger $C$ et al. CD19 CAR-T cells of defined CD4+:CD8+ composition in adult B cell ALL patients. J Clin Invest. 2016;126 (6):2123-2138.

26. Kebriaei P, Izsvák Z, Narayanavari SA, Singh H, Ivics Z. Gene Therapy with the Sleeping Beauty Transposon System. Trends Genet. 2017;33 (11):852-870.

27. Qasim W, Zhan H, Samarasinghe $S$ et al. Molecular remission of infant B-ALL after infusion of universal TALEN gene-edited CAR T cells. Sci Transl Med. 2017;9 (374

28. Torikai H, Reik A, Soldner F et al. Toward eliminating HLA class I expression to generate universal cells from allogeneic donors. Blood. 2013;122 (8):1341-1349.

29. Maude SL, Teachey DT, Rheingold SR et al. Sustained remissions with CD19-specific chimeric antigen receptor (CAR)-modified T cells in children with relapsed/refractory ALL. Journal of Clinical Oncology

JCO. 2016;34 (15_suppl):3011.

30. Lee DW, Kochenderfer JN, Stetler-Stevenson M et al. T cells expressing CD19 chimeric antigen receptors for acute lymphoblastic leukaemia in children and young adults: a phase 1 dose-escalation trial. The Lancet. 2015;385 (9967):517-528.

31. Park JH, Riviere I, Gonen M et al. Long-Term Follow-up of CD19 CAR Therapy in Acute Lymphoblastic Leukemia. N Engl J Med. 2018;378 (5):449-459.

32. Sotillo E, Barrett DM, Black KL et al. Convergence of Acquired Mutations and Alternative Splicing of CD19 Enables Resistance to CART-19 Immunotherapy. Cancer Discov. 2015

33. Neelapu SS, Tummala S, Kebriaei $\mathrm{P}$ et al. Chimeric antigen receptor T-cell therapy assessment and management of toxicities. Nat Rev Clin Oncol. 2018;15 (1):47-62.

34. Maude SL, Laetsch TW, Buechner J et al. Tisagenlecleucel in Children and Young Adults with B-Cell Lymphoblastic Leukemia. N Engl J Med. 2018;378 (5):439-448. 
35. Teachey DT, Lacey SF, Shaw PA et al. Identification of Predictive Biomarkers for Cytokine Release Syndrome after Chimeric Antigen Receptor T-cell Therapy for Acute Lymphoblastic Leukemia. Cancer Discov. 2016;6 664-679.

36. Wang $Z$, Han W. Biomarkers of cytokine release syndrome and neurotoxicity related to CAR-T cell therapy. Biomark Res. 2018;6 4.

37. Maude SL, Frey N, Shaw PA et al. Chimeric Antigen Receptor T Cells for Sustained Remissions in Leukemia. New England Journal of Medicine. 2014;371 (16):1507-1517.

38. Hay KA, Hanafi LA, Li D et al. Kinetics and Biomarkers of Severe Cytokine Release Syndrome after CD19 Chimeric Antigen Receptor-modified T Cell Therapy. Blood. 2017

39. Frey NV, Aplenc R, Barrett DM et al. T Cells Engineered With a Chimeric Antigen Receptor (CAR) Targeting CD19 (CTL019) Produce Significant In Vivo Proliferation, Complete Responses and Long-Term Persistence Without Gvhd In Children and Adults With Relapsed, Refractory ALL. Blood. 2013;122 (21):67-67.

40. Ghorashian S, Kramer AM, Albon SJ et al. A Novel Low Affinity CD19CAR Results in Durable Disease Remissions and Prolonged CAR T Cell Persistence without Severe CRS or Neurotoxicity in Patients with Paediatric ALL. Blood. 2017;130 (Suppl 1):806.

41. Lee DW, Gardner R, Porter DL et al. Current concepts in the diagnosis and management of cytokine release syndrome. Blood. 2014;124 (2):188-195.

42. Fitzgerald JC, Weiss SL, Maude SL et al. Cytokine Release Syndrome After Chimeric Antigen Receptor T Cell Therapy for Acute Lymphoblastic Leukemia. Crit Care Med. 2016

43. Stein AM, Grupp SA, Levine JE et al. CTL019 Model-Based Cellular Kinetic Analysis of Chimeric Antigen Receptor (CAR) T Cells to Characterize the Impact of Tocilizumab on Expansion and to Identify Correlates of Cytokine Release Syndrome Severity. Blood. 2017;130 (Suppl 1):2561.

44. Maude SL, Barrett D, Teachey DT, Grupp SA. Managing cytokine release syndrome associated with novel T cell-engaging therapies. Cancer J. 2014;20 (2):119-122.

45. Gust J, Hay KA, Hanafi LA et al. Endothelial Activation and Blood-Brain Barrier Disruption in Neurotoxicity after Adoptive Immunotherapy with CD19 CAR-T Cells. Cancer Discov. 2017

46. Hill JA, Li D, Hay KA et al. Infectious complications of CD19-targeted chimeric antigen receptor-modified T cell immunotherapy. Blood. 2017

47. Shalabi H, Shah NN, Fry TJ, Yates B, Delbrook C. Chimeric Antigen Receptor Induced Cytopenia Differs from Chemotherapy Induced Myelosuppression. Blood. 2017;130 (Suppl 1):5048.

48. Roberts $A D$, Ely $K H$, Woodland DL. Differential contributions of central and effector memory T cells to recall responses. J Exp Med. 2005;202 (1):123-133.

49. Wherry EJ, Teichgräber V, Becker TC et al. Lineage relationship and protective immunity of memory CD8 T cell subsets. Nat Immunol. 2003;4 (3):225-234.

50. Klebanoff CA, Gattinoni L, Torabi-Parizi $P$ et al. Central memory self/tumor-reactive CD8+ T cells confer superior antitumor immunity compared with effector memory $T$ cells. Proc Natl Acad Sci U S A. 2005;102 (27):9571-9576.

51. Gattinoni L, Lugli $E$, Ji $Y$ et al. A human memory $T$ cell subset with stem cell-like properties. Nat Med. 2011;17 (10):1290-1297.

52. $\mathrm{Xu} Y$, Zhang $\mathrm{M}$, Ramos $\mathrm{CA}$ et al. Closely related T-memory stem cells correlate with in vivo expansion of CAR.CD19-T cells and are preserved by IL-7 and IL-15. Blood. 2014;123 (24):3750-3759. 
53. Mock U, Nickolay L, Philip B et al. Automated manufacturing of chimeric antigen receptor T cells for adoptive immunotherapy using CliniMACS prodigy. Cytotherapy. 2016;18 (8):1002-1011.

54. Roddie C, Nickolay L, Mock U et al. Automated Manufacture of CAR19 Donor Lymphocytes Incorporated into an Escalated Dose DLI Protocol: Preliminary Results from the CARD Study. Blood. 2017;130 (Suppl 1):1993.

55. Singh N, Perazzelli J, Grupp SA, Barrett DM. Early memory phenotypes drive T cell proliferation in patients with pediatric malignancies. Sci Transl Med. 2016;8 (320):320ra3.

56. Guedan S, Posey ADJ, Shaw $C$ et al. Enhancing CAR T cell persistence through ICOS and 4-1BB costimulation. JCI Insight. 2018;3 (1

57. Gomes-Silva D, Mukherjee $M$, Srinivasan $M$ et al. Tonic 4-1BB Costimulation in Chimeric Antigen Receptors Impedes T Cell Survival and Is Vector-Dependent. Cell Rep. 2017;21 (1):17-26.

58. Wielgosz MM, Kim YS, Carney GG et al. Generation of a lentiviral vector producer cell clone for human Wiskott-Aldrich syndrome gene therapy. Mol Ther Methods Clin Dev. 2015;2 14063.

59. Sanber KS, Knight SB, Stephen SL et al. Construction of stable packaging cell lines for clinical lentiviral vector production. Sci Rep. 2015;5 9021.

60. Hacein-Bey-Abina S, Von Kalle C, Schmidt M et al. LMO2-associated clonal T cell proliferation in two patients after gene therapy for SCID-X1. Science. 2003;302 (5644):415419.

61. Howe SJ, Mansour MR, Schwarzwaelder K et al. Insertional mutagenesis combined with acquired somatic mutations causes leukemogenesis following gene therapy of SCID-X1 patients. J Clin Invest. 2008;118 (9):3143-3150.

62. Lacey SF, Xu J, Ruella M et al. Cars in Leukemia: Relapse with Antigen-Negative Leukemia Originating from a Single B Cell Expressing the Leukemia-Targeting CAR. Blood. 2016;128 (22):281.

63. Stroncek DF, Ren J, Lee DW et al. Myeloid cells in peripheral blood mononuclear cell concentrates inhibit the expansion of chimeric antigen receptor T cells. Cytotherapy. 2016

64. Brudno JN, Somerville RP, Shi V et al. Allogeneic T Cells That Express an Anti-CD19 Chimeric Antigen Receptor Induce Remissions of B-Cell Malignancies That Progress After Allogeneic Hematopoietic Stem-Cell Transplantation Without Causing Graft-Versus-Host Disease. J Clin Oncol. 2016;34 (10):1112-1121.

65. Cruz CR, Micklethwaite KP, Savoldo B et al. Infusion of donor-derived CD19-redirected virus-specific $T$ cells for B-cell malignancies relapsed after allogeneic stem cell transplant: a phase 1 study. Blood. 2013;122 (17):2965-2973.

66. Dai H, Wang Y, Lu X, Han W. Chimeric Antigen Receptors Modified T-Cells for Cancer Therapy. J Natl Cancer Inst. 2016;108 (7

67. Ghosh A, Smith M, James SE et al. Donor CD19 CAR T cells exert potent graft-versuslymphoma activity with diminished graft-versus-host activity. Nat Med. 2017

68. Pieters $R$, Schrappe $M$, De Lorenzo $P$ et al. A treatment protocol for infants younger than 1 year with acute lymphoblastic leukaemia (Interfant-99): an observational study and a multicentre randomised trial. Lancet. 2007;370 (9583):240-250.

69. Gardner R, Wu D, Cherian S et al. Acquisition of a CD19 negative myeloid phenotype allows immune escape of MLL-rearranged B-ALL from CD19 CAR-T cell therapy. Blood. 2016 70. Maude SL, Hucks GE, Callahan C et al. Durable Remissions with Humanized CD19Targeted Chimeric Antigen Receptor (CAR)-Modified T Cells in CAR-Naive and CAR-Exposed 
Children and Young Adults with Relapsed/Refractory Acute Lymphoblastic Leukemia. Blood. 2017;130 (Suppl 1):1319.

71. Shah NN, Highfill SL, Shalabi H et al. CD4/CD8 T-Cell Selection Enhances CD22 CAR-T Cell Transduction and in-Vivo CAR-T Expansion: Updated Results on Phase I Anti-CD22 CAR Dose Expansion Cohort. Blood. 2017;130 (Suppl 1):809.

72. Fry TJ, Shah NN, Orentas RJ et al. CD22-targeted CAR T cells induce remission in B-ALL that is naive or resistant to CD19-targeted CAR immunotherapy. Nat Med. 2018;24 (1):20-28. 73. Eshhar Z, Waks T, Gross G, Schindler DG. Specific activation and targeting of cytotoxic lymphocytes through chimeric single chains consisting of antibody-binding domains and the gamma or zeta subunits of the immunoglobulin and T-cell receptors. Proc Natl Acad Sci U S A. 1993;90 (2):720-724.

74. Davila ML, Riviere I, Wang X et al. Efficacy and toxicity management of 19-28z CAR T cell therapy in B cell acute lymphoblastic leukemia. Sci Transl Med. 2014;6 (224):224ra25.

75. Lee DW, Stetler-Stevenson M, Yuan CM et al. Long-Term Outcomes Following CD19 CAR T Cell Therapy for B-ALL Are Superior in Patients Receiving a Fludarabine/Cyclophosphamide Preparative Regimen and Post-CAR Hematopoietic Stem Cell Transplantation. Blood. 2016;128 (22):218.

76. Dai H, Zhang W, Li X et al. Tolerance and efficacy of autologous or donor-derived T cells expressing CD19 chimeric antigen receptors in adult B-ALL with extramedullary leukemia. Oncoimmunology. 2015;4 (11):e1027469.

77. Kebriaei $\mathrm{P}$, Singh $\mathrm{H}$, Huls $\mathrm{MH}$ et al. Phase I trials using Sleeping Beauty to generate CD19-specific CAR T cells. J Clin Invest. 2016

\section{Acknowledgements}

All research at Great Ormond Street Hospital NHS Foundation Trust and UCL Great Ormond Street Institute of Child Health is made possible by the NIHR Great Ormond Street Hospital Biomedical Research Centre. The views expressed are those of the author(s) and not necessarily those of the NHS, the NIHR or the Department of Health.

Conflicting Interests Statement

Sara Ghorashian

UCL: Patents \& Royalties: UCL Business

Honoraria: Novartis, Travel Sponsorship: Celgene

Persis Amrolia

UCL: Patents \& Royalties: UCL Business

Paul Veys

Honoraria: Chimerix, EUSA, Novartis, Gilead, and Jazz pharmaceuticals.

Funding statement:

Persis Amrolia receives research funding from UK Medical Research Council, EU Horizon2020 programme, GOSH Children's Charity, Children with Leukaemia, Bluebirdbio and is a recipient of an NIHR Research Professorship. 
Paul Veys receives research funding from Servier and from Bellicum Pharmaceuticals 